\title{
Marked colimits and higher cofinality
}

\author{
Fernando Abellán García ${ }^{1}$
}

Received: 22 October 2020 / Accepted: 2 November 2021 / Published online: 16 December 2021

(c) The Author(s) 2021

\begin{abstract}
Given a marked $\infty$-category $\mathcal{D}^{\dagger}$ (i.e. an $\infty$-category equipped with a specified collection of morphisms) and a functor $F: \mathcal{D} \rightarrow \mathbb{B}$ with values in an $\infty$-bicategory, we define $\operatorname{colim}^{\dagger} F$, the marked colimit of $F$. We provide a definition of weighted colimits in $\infty$-bicategories when the indexing diagram is an $\infty$-category and show that they can be computed in terms of marked colimits. In the maximally marked case $\mathcal{D}^{\sharp}$, our construction retrieves the $\infty$-categorical colimit of $F$ in the underlying $\infty$-category $\mathcal{B} \subseteq \mathbb{B}$. In the specific case when $\mathbb{B}=\mathfrak{l a t}_{\infty}$, the $\infty$-bicategory of $\infty$ categories and $\mathcal{D}^{b}$ is minimally marked, we recover the definition of lax colimit of Gepner-Haugseng-Nikolaus. We show that a suitable $\infty$-localization of the associated coCartesian fibration $\operatorname{Un}_{\mathcal{D}}(F)$ computes colim ${ }^{\dagger} F$. Our main theorem is a characterization of those functors of marked $\infty$-categories $f: \mathcal{C}^{\dagger} \rightarrow \mathcal{D}^{\dagger}$ which are marked cofinal. More precisely, we provide sufficient and necessary criteria for the restriction of diagrams along $f$ to preserve marked colimits
\end{abstract}

Keywords Infinity bicategories · Weighted colimits · Cofinality · localization

\section{Introduction}

The theory of $\infty$-categories is, by now, well-established as an excellent way to treat coherence and higher homotopical data. However, the mere presence of this higher data means that many properties which can be explored most easily by explicit computation in the ordinary categorical setting are best accessed by universal properties in the $\infty$-categorical setting. Consequently, general constructions exhibiting universal properties take on an even greater importance in the study of higher category theory. Among such constructions, the theories of limits and colimits form an essential core around which many of the results in higher category theory are built. In the developing

Communicated by Emily Riehl.

$\bowtie \quad$ Fernando Abellán García

fernando.abellan@uni-hamburg.de

1 Fachbereich Mathematik, Universität Hamburg, Bundesstraße 55, 20146 Hamburg, Germany 
theory of $\infty$-bicategories, as in its strict 1-categorical analogue, these theories must be extended to allow for laxness - loosely, allowing cones over a functor to commute up to non-invertible 2-morphism.

This paper is a new entry in this story, dealing with lax colimits which depend on a collection of marked morphisms in the source. These morphisms can be viewed as "controlling the laxness" of the colimit in question. Such collections of marked morphisms arise throughout the study of higher categories - in localizations, cartesian fibrations, etc. The theory of marked colimits and marked cofinality developed in this paper represents a new technology for treating such objects. Along the way to $\infty$ cofinality, we will also see that the theory of weighted colimits expounded in [6] can be viewed as one instance of the general theory of marked colimits, and will note a fundamental relation to the Grothendieck construction, generalizing extant results for lax colimits and usual $\infty$-colimits.

A comment from the author (22/06/20): After completion of this work, a prepint [4] appeared proving Theorem 3 below. This result was achieved independently by both authors using completely different methods of proof. In this work, the definition of marked colimit provided is characterized by a 2-dimensional universal property. By the time the paper [4] was uploaded I was working in the proof of Proposition 2 in order to show that the definition of weighted colimits appearing in [6] satisfies this 2-dimensional universal property. The reader not willing to take Proposition 2 on faith can adapt the proof to the definition of lax colimit provided in the aforementioned papers.

Update (28/09/20): The proof of Proposition 2 can be now found in [2].

Marked colimits in 2-categories. Let $F: \mathbb{C} \rightarrow \mathbb{B}$ be a 2-functor. A lax cone for $F$ with vertex point $b \in \mathbb{B}$ is given by the following data

- For every object $c \in C$ a morphism $\alpha_{c}: F(c) \rightarrow b$.

- For every morphism $u: c \rightarrow c^{\prime}$ in $C$ a 2-morphism $\theta_{u}: \alpha_{c} \Rightarrow \alpha_{c^{\prime}} \circ F(u)$ depicted by a 2-commutative diagram

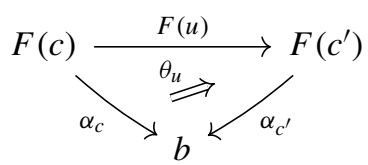

These data must satisfy the following set of axioms

(I) Unitality: $\theta_{\mathrm{id}_{c}}=\mathrm{id}_{\alpha_{c}}$ for every $c \in \mathbb{C}$. (II) Composability: Given $u: c \rightarrow c^{\prime}$ and $v: c^{\prime} \rightarrow c^{\prime \prime}$ the following equation holds

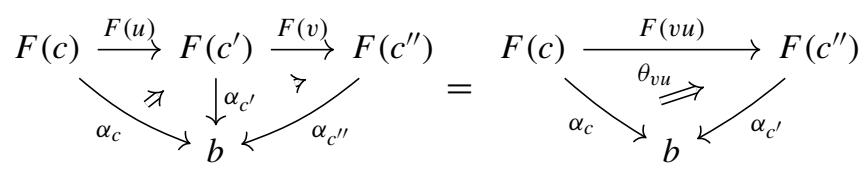

(III) Compatibility with 2-morphisms: For every 2-morphism $\eta: u \Rightarrow u^{\prime}$ in $\mathbb{C}$ the following equation holds 


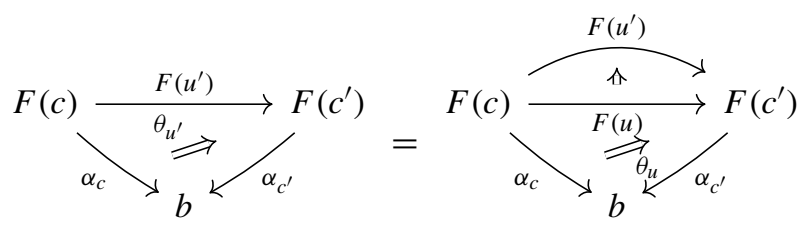

A morphism of lax cones $\left\{\alpha_{c}\right\}_{c \in C} \rightarrow\left\{\beta_{c}\right\}_{c \in C}$ with vertex point $b$ is given by a family of 2-morphisms $\left\{\epsilon_{c}: \alpha_{c} \Rightarrow \beta_{c}\right\}_{c \in C}$ such that for every $f: c \rightarrow c^{\prime}$ the following equation holds

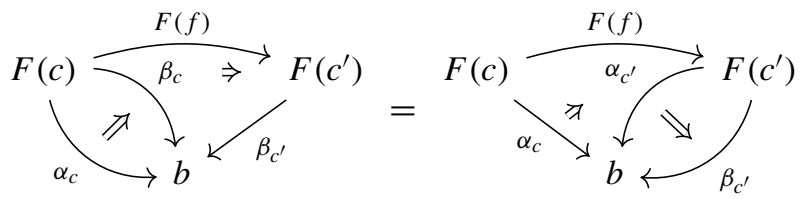

One can then show that this defines a category of lax cones for $F$ with vertex $b$. Furthermore, we can arrange the previous definitions into a 2-functor with values in the 2-category of categories

$$
\mathbb{B} \rightarrow \mathbb{C} \text { at } ; b \mapsto\{\text { category lax cones with vertex } b \text { \} }
$$

The laxcolimit of $F$ is then defined as an object $\operatorname{colim}^{[}{ }_{C} F \in \mathbb{B}$ correpresenting the functor above (see [7] for a classical reference on lax limits). In practice it is common to work with a category $C$ (resp. 2-category $\mathbb{C}$ ) equipped with a collection of chosen morphisms containing the identities (marked categories, resp. marked 2-categories) that one wants to formally invert. It is thus desirable to have a theory of colimits adapted to accomodate the extra information present in marked (2)-categories.

Let $\mathbb{C}^{\dagger}$ be a marked 2-category. We define a marked cone for $F$ to be a lax cone such that the 2-morphisms $\theta_{f}$ are invertible whenever $f$ is a marked morphism in $\mathbb{C}^{\dagger}$. Paralleling the construction above, we obtain the notion of the marked colimit of $F$. This definition was already present in the literature under the name of $\sigma$-colimits [5], where it was succesfully applied to define a 2-dimensional theory of flat pseudofunctors. Although similar in spirit, our definition of marked colimits presented in Sect. 3 will depart from that of Descotte et al. The main cause of this difference is our use of weighted colimits as the jumping-off point of our theory, rather than using different levels of laxness (see Definition 2.4.3 in [5]) in the natural transformation defining a marked cone. We have opted for this approach since to our eyes is the one that is better suited for developing the cofinality theory that we will now introduce.

Marked colimits in $\infty$-bicategories. Let $\mathfrak{l} a t_{\infty}$ denote the $\infty$-bicategory of $\infty$ categories. Let $F: \mathcal{C} \rightarrow \mathbb{B}$ and $W: \mathcal{C}^{\text {op }} \rightarrow \mathfrak{l}^{2} t_{\infty}$ be functors where $\mathcal{C}$ is an $\infty$-category and $\mathbb{B}$ is an $\infty$-bicategory. In Sect. 3 we define $W \otimes F$, the colimit of $F$ weighted by $W$. Once this basic theory is established we embark upon the main construction of this work, the definition of marked colimits, appearing in Sect. 4. Given a marked 
$\infty$-category $\mathrm{C}^{\dagger}$ we define a functor

$$
\mathfrak{C}_{\mathcal{C} /}^{\dagger}: \mathcal{C}^{\text {op }} \rightarrow \mathfrak{C a t}_{\infty} ; c \mapsto \mathrm{L}_{W}\left(\mathrm{C}_{c /}^{\dagger}\right)
$$

where $\mathrm{L}_{W}\left(\mathrm{C}_{c /}^{\dagger}\right)$ stands for the $\infty$-localization of the slice category $\mathrm{C}_{c /}$ with respect to the marking induced by $\mathrm{C}^{\dagger}$. This allows us to define the marked colimit of a functor $F: \mathcal{C} \rightarrow \mathbb{B}$ as $\mathfrak{C}_{\mathcal{C} /}^{\dagger} \otimes F$. The definition presented naturally extends the notion of $\infty$ colimits appearing in $[3,10]$, as demonstrated by the following result.

Theorem 1 Let $F: \mathcal{C} \rightarrow \mathbb{B}$ and suppose that colim ${ }^{3} \mathrm{C} F$ exists. Then the $\infty$-categorical colimit of $F$ in the underlying $\infty$-category $\mathcal{B} \subseteq \mathbb{B}$ exists and there is an equivalence

$$
\operatorname{colim}_{\mathrm{e}} \mathrm{F} \stackrel{\simeq}{\rightarrow} \operatorname{colim}_{\mathrm{e}} F
$$

In particular, both universal properties coincide if $\mathbb{B}=\mathcal{B}$.

The rest of Sect. 4 is devoted to computational results. First, we show that weighted colimits indexed by an $\infty$-category $\mathcal{C}$ can be computed in terms of marked colimits. Let $W: \mathrm{Cop}^{\rightarrow} \mathfrak{L a t}_{\infty}$ be a weight functor. Let us observe that its associated Cartesian fibration $\mathcal{W}$ comes equipped with a canonical marking given by the Cartesian edges. We denote this marked category by $\mathcal{W}^{\natural}$. With the aid of this observation, we can then prove:

Theorem 2 Let $F: \mathcal{C} \rightarrow \mathbb{B}$ and $W: e^{o p} \rightarrow \mathfrak{C a t}_{\infty}$. Let $p: \mathcal{W} \rightarrow \mathcal{C}$ denote the Cartesian fibration classifying $W$. Suppose that $W \otimes F$ exists. Then $\operatorname{colim}^{\prime} \mathcal{W} F \circ p$ exists and there is an equivalence in $\mathbb{B}$

$$
W \otimes F \stackrel{\simeq}{\longrightarrow} \operatorname{colim}_{W}^{\natural} F \circ p
$$

We conclude Sect. 4 by providing a thorough analysis of marked colimits in $\mathbb{L} a t_{\infty}$. To achieve this goal, we will employ the Grothendieck construction in its $\infty$-categorical incarnation as the unstraightening functor (see chapter 3 in [10]), as the "universal recipe" to compute colimits of diagrams in $\infty$-categories. This is witnessed by two results already known in the literature: in [6] the authors define lax colimits in $\infty$ categories and show in Theorem 7.4 that given a functor $F$ with values in $\infty$-categories its associated coCartesian fibration computes the lax colimit. Let us remark that our definition in the minimally marked case $\mathcal{C}^{b}$ particularizes to that of Gepner et al. If we focus our attention on the maximally marked case $\mathcal{C}^{\sharp}$, we note that Corollary 3.3.4 in [10] shows that the colimit of a functor $F$ with values in $\infty$-categories can be computed as the $\infty$-localization of its associated coCartesian fibration at the collection of coCartesian edges.

Therefore, we observe that the two extremal cases $\mathcal{C}^{b}$ resp. $\mathcal{C}^{\sharp}$, are already well understood. It is then natural to ask ourselves if there is a generalization of both results which can be seen through the lens of marked colimits. We provide an affirmative 
answer to this question in the following result which can be found as Theorem 8 in the main body of this article.

Theorem 3 Let $\mathcal{C}^{\dagger}$ be a marked $\infty$-category. Given $F: \mathcal{C} \rightarrow \mathfrak{C a t}_{\infty}$ there is a equivalence of $\infty$-categories

$$
\mathrm{L}_{W}\left(\mathrm{Un}_{\mathcal{C}}^{\mathrm{co}}(F)^{\natural(\dagger)}\right) \simeq \operatorname{colim}^{\dagger} \mathrm{e} F
$$

where $\mathrm{L}_{W}\left(\mathrm{Un}_{\mathcal{C}}^{\mathrm{co}}(F)^{\natural(\dagger)}\right)$ denotes the $\infty$-localization at the collection of coCartesian edges lying over marked edges of $\mathrm{C}^{\dagger}$.

A cofinality criterion: Theorem $\mathbf{A}^{\dagger}$. Let $f: \mathcal{C}^{\dagger} \rightarrow \mathcal{D}^{\dagger}$ be a marking-preserving functor. We call $f$ a marked cofinal functor if for every diagram $F: \mathcal{D} \rightarrow \mathbb{B}$ the canonical comparison map ${ }^{1}$

$$
\operatorname{colim}^{\dagger} \mathrm{e} F \circ f \stackrel{\simeq}{\rightarrow} \operatorname{colim}^{\dagger} \mathcal{D} F
$$

is an equivalence in $\mathbb{B}$. The main result of this work is a characterization of this higher notion of cofinality.

Theorem 4 Let $f: \mathcal{C}^{\dagger} \rightarrow \mathcal{D}^{\dagger}$ be a marking-preserving functor of $\infty$-categories. Then $f$ is marked cofinal if and only if for every $d \in \mathcal{D}$ the canonical map $\mathcal{C}_{d /}^{\dagger} \rightarrow \mathcal{D}_{d /}^{\dagger}$ induces an equivalence on localized $\infty$-categories,

$$
\mathrm{L}_{W}\left(\mathrm{C}_{d /}^{\dagger}\right) \stackrel{\simeq}{\rightarrow} \mathrm{L}_{W}\left(\mathcal{D}_{d /}^{\dagger}\right)
$$

As a corollary we obtain a generalization of Theorem A in [12] to marked $\infty$ categories.

Corollary 1 (Theorem $\mathrm{A}^{\dagger}$ ) Let $f: \mathrm{C}^{\dagger} \rightarrow \mathcal{D}^{\dagger}$ be a marking-preserving functor of $\infty$ categories. If the canonical map $\mathrm{L}_{W}\left(\mathcal{C}_{d /}^{\dagger}\right) \stackrel{\simeq}{\rightarrow} \mathrm{L}_{W}\left(\mathcal{D}_{d /}^{\dagger}\right)$ is an equivalence of $\infty$ categories for every $d \in \mathcal{D}$, then the induced functor on $\infty$-localizations

$$
f_{W}: \mathrm{L}_{W}\left(\mathrm{C}^{\dagger}\right) \stackrel{\simeq}{\rightarrow} \mathrm{L}_{W}\left(\mathcal{D}^{\dagger}\right)
$$

is an equivalence of $\infty$-categories.

In previous work, (see [1] for general background and notation) we generalized Quillen's Theorem A to (strict) marked 2-categories.

Theorem 5 Let $F: \mathbb{C}^{\dagger} \rightarrow \mathbb{D}^{\dagger}$ be a functor of marked 2-categories. Suppose that,

1. For every object $d \in \mathbb{D}$, there exists a morphism $g_{d}: d \rightarrow F(c)$ which is initial in both $\mathrm{L}_{W}\left(\mathbb{C}_{d \downarrow}^{\dagger}\right)$ and $\mathrm{L}_{W}\left(\mathbb{D}_{d \downarrow}^{\dagger}\right)$.

\footnotetext{
1 See Sect.5 for a precise definition of the comparison map.
} 
2. Every marked morphism $d \rightarrow F(c)$ is initial in $\mathrm{L}_{W}\left(\mathbb{C}_{d \downarrow}^{\dagger}\right)$.

3. For any marked morphism

$f: b \rightarrow d$ in $\mathbb{D}$, the induced functors $f^{*}: \mathrm{L}_{W}\left(\mathbb{C}_{d \downarrow}^{\dagger}\right) \rightarrow \mathrm{L}_{W}\left(\mathbb{C}_{b \downarrow}^{\dagger}\right)$ preserve initial objects.

Then the induced functor $F_{W}: \mathrm{L}_{W}\left(\mathrm{~N}_{2}\left(\mathbb{C}^{\dagger}\right)\right) \rightarrow \mathrm{L}_{W}\left(\mathrm{~N}_{2}\left(\mathbb{D}^{\dagger}\right)\right)$ is an equivalence of $\infty$-categories.

We also claimed that the conditions of the previous theorem should control the notion of higher cofinality for $\infty$-bicategories. This work can be understood as a partial result towards proving the cofinality conjecture as stated in [1]. This will be justified in Theorem 9 where we show that the hypotheses of Theorem 4 are equivalent to the analogous conditions of Theorem 5 for $\infty$-categories.

The importance of Theorem 4 becomes apparent when combined with Theorem 2. The use of weighted colimits permeates throughout category theory. Many essential constructions, for example, Kan extensions, are best formulated in terms of weighted (co)limits. However, to the author's knowledge the general theory was lacking tools that allow us to simplify the computations of weighted colimits. Marked colimits are devised as an equivalent language to that of their weighted counterparts that allows for transparent cofinality statements. Let us consider for example a functor $f: \mathcal{C} \rightarrow \mathcal{D}$ between $\infty$-categories. A common situation that we might encounter ourselves in is to try to determine whether the restriction functor

$$
f^{*}: \operatorname{Fun}\left(\mathcal{D}, \mathcal{C a t}_{\infty}\right) \rightarrow \operatorname{Fun}\left(\mathcal{C}, \mathcal{C a t}_{\infty}\right)
$$

is fully faithful. An elegant sufficient criterion to check is to show that for every $d \in \mathcal{D}$ the induced morphism is $\mathfrak{C}_{d /} \rightarrow \mathcal{D}_{d /}$ is cofinal in the usual sense. Once our cofinality theory is developed to its full 2-category potential such statements will be available to the public allowing a better access to complicated constructions such as functor $\infty$-bicategories.

\section{Preliminaries}

In this section, we collect notation, definitions and background necessary for our constructions and proofs. We assume basic familiarity with the theory of $\infty$-categories as in $[3,10]$. We will use scaled simplicial sets as a model for $\infty$-bicategories following [9]. We refer the reader to [8] for the basics of enriched category theory including weighted (co)limits.

Notation 1 We will denote ordinary strict 1-categories by capital letters $(A, B, C)$ and $\infty$-categories by caligraphic letters $(\mathcal{A}, \mathcal{B}, \mathcal{C})$. We will generally (see Remark 1 below for an exception) denote $\infty$-bicategories by boldface letters $(\mathbb{A}, \mathbb{B}, \mathbb{C})$.

Remark 1 Following the previous convention we will denote by Cat $t_{\infty}$ the full subcategory of the 1-category of simplicial sets consisting of $\infty$-categories. We will use 
the notation $\mathcal{C}_{\infty}$ to denote the $\infty$-category of $\infty$-categories. We will denote the $\infty$-bicategory of $\infty$-categories by $\mathfrak{l a t}_{\infty}$.

Notation 2 We will extensively use marked simplicial sets as a model for $\infty$ categories. Given a simplicial set $X$ we denote by $X^{b} \in \operatorname{Set}_{\Delta}^{+}$the marked simplicial set with only degenerate edges marked and by $X^{\sharp}$ the marked simplicial set with all edges marked.

Notation 3 Given a simplicial set $X$ we denote by $X_{b} \in \operatorname{Set}_{\Delta}^{\text {sc }}$ the scaled simplicial set with only degenerate 2 -simplices being thin and by $X_{\sharp}$ the scaled simplicial set with all 2-simplices being thin. We will identify $\infty$-categories with maximally scaled simplicial sets that are fibrant in the bicategorical model structure.

Notation 4 Given an $\infty$-bicategory $\mathbb{B}$ and objects $x, y \in \mathbb{B}$, we denote by $\mathbb{B}(x, y) \in$ $\mathfrak{L} \mathfrak{a t}_{\infty}$ the mapping category. For every $y \in \mathbb{B}$ we denote by $\mathbb{B}(-, y)$ the functor

$$
\mathbb{B}^{\mathrm{op}} \rightarrow \mathfrak{C a t}_{\infty} ; x \mapsto \mathbb{B}(x, y) .
$$

Notation 5 Given $\infty$-bicategories $\mathbb{B}, \mathbb{C}$ and functors $F, G: \mathbb{C} \rightarrow \mathbb{B}$ we denote by $\operatorname{Nat}_{\mathbb{B}}(F, G)$ the mapping category of Fun $(\mathbb{C}, \mathbb{B})$. We denote by $\operatorname{Nat}_{\mathbb{B}}(F, G)$ the underlying Kan complex.

Notation 6 Given an $\infty$-bicategory $\mathbb{B}$ we denote by $\mathcal{Y}_{\mathbb{B}}$ the $\infty$-bicategorical Yoneda embedding

$$
\mathbb{B} \rightarrow \operatorname{Fun}\left(\mathbb{B}^{\text {op }}, \mathfrak{C a t}_{\infty}\right) ; y \mapsto \mathbb{B}(-, y)
$$

Notation 7 Given a functor $F: \mathcal{C} \rightarrow \mathcal{C}_{\infty}$ we will denote by $\operatorname{Un}_{\mathcal{C}}^{\text {co }}(F)$ the value of the (coCartesian) unstraightening functor as defined (with the adequate dualization) in 3.2.1 [10]. Since $U n_{\mathcal{C}}^{c o}$ induces an equivalence of $\infty$-categories between Fun $\left(\mathcal{C}, \mathcal{C a t}_{\infty}\right)$ and the $\infty$-category of coCartesian fibrations over $\mathcal{C}$ we will often call $\operatorname{Un}_{\mathcal{C}}^{\mathrm{co}}(F)$ the "coCartersian fibration associated to $F$ ".

\subsection{Marked $\infty$-categories and localizations}

Definition 1 Let $U: \operatorname{Set}_{\Delta}^{+} \rightarrow \operatorname{Set}_{\Delta}$ be the forgetful functor. We define $\mathrm{Cat}_{\infty}^{\dagger}$ (see Remark 1) as the full subcategory of $\operatorname{Set}_{\Delta}^{+}$on those objects $X^{\dagger} \in \operatorname{Set}_{\Delta}^{+}$such that $U\left(X^{\dagger}\right)$ is an $\infty$-category. We call the objects of Cat ${ }_{\infty}^{\dagger}$ marked $\infty$-categories and its morphisms marked functors.

Definition 2 Let $f: \mathcal{C}^{\dagger} \rightarrow \mathcal{D}^{\dagger}$ be a marked functor. Given $d \in \mathcal{D}$ we define a marking on $\mathcal{C}_{d}$ / by declaring an edge $\sigma: \Delta^{1} \rightarrow \mathcal{C}_{d}$ to be marked if an only if its image under the canonical map is marked in $\mathcal{C}^{\dagger}$. We denote this marked simplicial set by $\mathcal{C}_{d /}^{\dagger} \in \operatorname{Set}_{\Delta}^{+}$.

Notation 8 Given a marked $\infty$-category $\mathcal{C}^{\dagger}$ we denote its $\infty$-categorical localization with respect to its marked edges by $\mathrm{L}_{W}\left(\mathcal{C}^{\dagger}\right)$. Given $X \in \mathcal{C a t}_{\infty}$ we define Fun ${ }^{\dagger}(\mathcal{C}, X)$ to be the full subcategory of Fun $(\mathcal{C}, \mathcal{X})$ on those functors mapping marked edges of $\mathcal{C}$ to equivalences in $X$. 
Remark 2 Note that the universal property of localizations implies that we have an equivalence of $\infty$-categories $\operatorname{Fun}^{\dagger}(\mathcal{C}, X) \simeq$ Fun $\left(\mathrm{L}_{W}\left(\mathcal{C}^{\dagger}\right), X\right)$. We remind the reader that a model for the localization of $\mathrm{C}^{\dagger}$ is given by its fibrant replacement in the model structure on marked simplicial sets.

\subsection{Free fibrations}

In this section, we review the main results of [6] regarding free Cartesian fibrations and their relation to marked $\infty$-categories.

Definition 3 Given $\mathcal{C} \in \mathcal{C a t}_{\infty}$ let $\mathcal{C a t}_{\infty / \mathcal{C}}^{\text {cart }}$ be the subcategory of the undercategory $\mathrm{Cat}_{\infty / \mathcal{C}}$, whose objects are Cartesian fibrations, and whose morphisms are functors which preserve Cartesian morphisms.

Remark 3 There is an obvious forgetful functor $\mathfrak{U}: \operatorname{Cat}_{\infty / \mathcal{C}}^{\mathrm{cart}} \rightarrow \mathcal{C a t}_{\infty / \mathcal{C}}$.

Notation 9 Given an $\infty$-category $\mathcal{C}$ and two Cartesian fibrations

$$
x \rightarrow \mathcal{C}, y \rightarrow \mathcal{C}
$$

we denote by Fun ${ }_{\mathcal{C}}^{\text {cart }}(X, y)$ the full subcategory of functors over $\mathcal{C}$ that preserve Cartesian morphisms.

Definition 4 Let $\mathcal{C}$ be an $\infty$-category. For $p: \mathcal{E} \rightarrow \mathcal{C}$ any functor of $\infty$-categories, let $\mathfrak{F}(p): \mathfrak{F}(\mathcal{E}) \rightarrow \mathcal{C}$ denote the map $\mathcal{E} \times \mathcal{C} \mathcal{C}^{\Delta^{1}} \rightarrow \mathcal{C}$, where the pullback is along the target fibration $\mathcal{C}^{\Delta^{1}} \rightarrow \mathcal{C}$ given by evaluation at $1 \in \Delta^{1}$, and the projection $\mathfrak{F}(p)$ is induced by evaluation at 0 . We call the projection map $\mathcal{E} \times \mathcal{e} \mathcal{C}^{\Delta^{1}} \rightarrow \mathcal{C}$ the free Cartesian fibration on $p$. The Cartesian edges of $\mathfrak{F}(p)$ are precisely those which are mapped to equivalences under the projection to $\mathcal{E}$. Then $\mathfrak{F}$ defines a functor

$$
\mathfrak{F}: \mathrm{Cat}_{\infty / \mathcal{C}} \rightarrow \mathrm{Cat}_{\infty / \mathcal{C}}^{\mathrm{cart}}
$$

Remark 4 Let $p: \mathcal{E} \rightarrow \mathcal{C}$. Then the objects of $\mathfrak{F}(\mathcal{E})$ are given by edges of $\mathcal{C}$ of the form $c \rightarrow p(e)$ where $e \in \mathcal{E}$. A morphism is given by a commutative diagram in $\mathcal{C}$

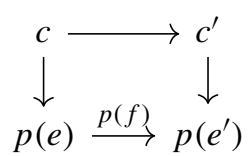

where $f$ is an morphism in $\mathcal{E}$.

Remark 5 Composition with the degeneracy map $s_{0}: \Delta^{1} \rightarrow \Delta^{0}$ induces a functor $\mathcal{C} \rightarrow \mathcal{C}^{\Delta^{1}}$ which is a section to both of the evaluation maps. Given a functor $\mathcal{E} \rightarrow \mathcal{C}$, this section gives a natural map

$$
\mathcal{E} \rightarrow \mathcal{E} \times \mathrm{e}^{\mathrm{e}^{\Delta^{1}}}
$$


over $\mathcal{C}$, inducing a unit natural transformation $\eta:$ id $\Rightarrow \mathfrak{U} \circ \mathfrak{F}$.

Proposition 1 Given a map of $\infty$-categories $\mathcal{E} \rightarrow \mathcal{C}$ then the unit natural transformation $\mathcal{E} \rightarrow \mathfrak{F}(\mathcal{E})$ induces an equivalence of $\infty$-categories

$$
\operatorname{Fun}_{\mathcal{C}}^{\text {cart }}(\mathfrak{F}(\mathcal{E}), \mathcal{X}) \rightarrow[\simeq] \operatorname{Fun}_{\mathcal{C}}(\mathcal{E}, \mathfrak{U}(\mathcal{X}))
$$

Proof This is Proposition 4.11 in [6].

Definition 5 Let $p: \mathcal{E} \rightarrow \mathcal{C}$ be a functor of $\infty$-categories. Suppose that $\mathcal{E}$ is a marked $\infty$-category and denote it by $\mathcal{E}^{\dagger}$. We declare an edge of $\mathcal{E} \times \mathrm{e} \mathrm{e}^{\Delta^{1}}$ to be marked if and only if its projection to $\mathcal{E}$ is marked. We denote this marked $\infty$-category over $\mathcal{C}$ by $\mathfrak{F}(\mathcal{E})^{\dagger}$.

Remark 6 Observe that in the previous definition, we can identify the fibers $\mathfrak{F}(\mathcal{E})^{\dagger} \times\{c\}$ $\mathcal{C}$ with the marked slice $\varepsilon_{c \text { / }}^{\dagger}$ where we declare an edge marked if and only if it is marked in $\mathcal{E}^{\dagger}$.

Definition 6 Let $p: \mathcal{E} \rightarrow \mathcal{C}$ be a functor of $\infty$-categories and assume further that $\mathcal{E}$ is a marked $\infty$-category. Given a Cartesian fibration $X \rightarrow \mathcal{C}$ we define $\operatorname{Fun}_{\mathcal{C}}^{\dagger}(\mathcal{E}, \mathcal{X})$ to be the full subcategory on those functors mapping marked edges in $\mathcal{E}^{\dagger}$ to Cartesian morphisms in $X$. If $p$ is a Cartesian fibration we define $\operatorname{Fun}_{\mathcal{C}}^{\text {cart }, \dagger}(\mathcal{E}, \mathcal{X})$ to be the full subcategory of $\operatorname{Fun}_{\mathcal{C}}^{\dagger}(\mathcal{E}, X)$ on those functors which also preserve the Cartesian edges of $\mathcal{E}$.

Lemma 1 Let $\mathcal{E}^{\dagger}$ be a marked $\infty$-category together with a functor $p: \mathcal{E} \rightarrow \mathcal{C}$. Consider $\mathfrak{F}(\mathcal{E})^{\dagger}$ as in Definition 5. Then the unit map $\eta$ induces a commutative diagram in $\mathrm{Cat}_{\infty}$

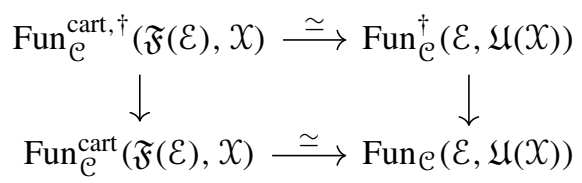

where the vertical maps are fully faithful and the horizontal maps are equivalences of $\infty$-categories.

Proof The vertical maps are fully faithful by definition. Since the bottom horizontal map is an equivalence by Proposition 1 it will suffice to compute its essential image when restricted to Fun $_{\mathcal{C}}^{\text {cart, } \dagger}(\mathfrak{F}(\mathcal{E}), X)$. It is clear that the image of this restricted map lands in $\operatorname{Fun}_{\mathcal{C}}^{\dagger}(\mathcal{E}, \mathfrak{U}(\mathcal{X}))$. Now suppose that we are given a functor of Cartesian fibrations $L: \mathfrak{F}(\mathcal{E}) \rightarrow X$ such that its image under the unit map lands in $\operatorname{Fun}_{\mathcal{C}}^{\dagger}(\mathcal{E}, \mathfrak{U}(X))$. Consider a marked edge in $\sigma: \Delta^{1} \rightarrow \mathfrak{F}(\mathcal{E})$ represented by a commutative diagram

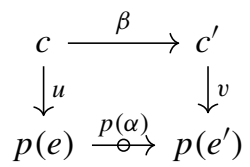


where $\alpha: \Delta^{1} \rightarrow \mathcal{E}$ is a marked morphism. We claim that $L(\sigma)$ is a Cartesian edge of $X$. First we observe that we have an inner horn $\theta: \Lambda_{1}^{2} \rightarrow X$ given by

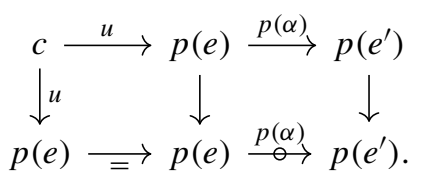

Using Proposition 2.4.1.7 in [10] we see that since both edges of the horn are Cartesian in $X$ it follows that any composite of those two edges must be Cartesian in $\chi$. We consider another horn $\Xi: \Lambda_{1}^{2} \rightarrow X$

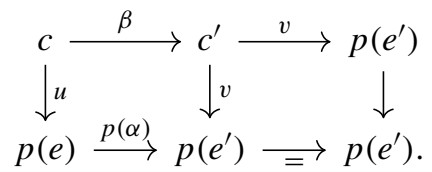

First we observe that the restriction $\Delta^{\{1,2\}} \rightarrow \Lambda_{2}^{1} \rightarrow[\Xi] X$ is a Cartesian edge and that the restriction of $\Xi$ to $\Delta^{\{0,1\}}$ is $L(\sigma)$. Finally one notes that any composite of the morphisms in $\theta$ must be homotopic to any composite of the morphisms in $\Xi$. Again by Proposition 2.4.1.7 in [10] this implies that $L(\sigma)$ is Cartesian in $X$.

Definition 7 Let $\pi: X \rightarrow \mathcal{C}$ be a Cartesian fibration and assume that $\mathcal{X}^{\dagger}$ is a marked $\infty$-category. A fiberwise localization of $\pi$ at the collection of marked edges of $X$ is a Cartesian fibration $\mathrm{L}_{W}^{\mathrm{e}}\left(\pi^{\dagger}\right) \rightarrow \mathcal{C}$ together with a map of Cartesian fibrations $\iota: X \rightarrow \mathrm{L}_{W}^{\mathrm{e}}\left(\pi^{\dagger}\right)$ such that

- The map $\iota$ sends marked edges in $\mathcal{X}$ to Cartesian edges in $\mathrm{L}_{W}^{\stackrel{C}{ }}\left(\pi^{\dagger}\right)$.

- For any Cartesian fibration $\mathcal{Y} \rightarrow \mathcal{C}$ the induced functor Fun $_{\mathcal{C}}^{\text {cart }}\left(\mathrm{L}_{W}^{\mathcal{E}}\left(\pi^{\dagger}\right), y\right) \rightarrow$ Fun $_{\mathcal{C}}^{\text {cart, } \dagger}(\mathcal{X}, y)$ is an equivalence of $\infty$-categories.

Remark 7 Given $\pi: X \rightarrow \mathcal{C}$ as above let $X^{\diamond}$ denote the marked simplicial set over $\mathcal{C}$ where an edge is marked if it is Cartesian or if it is marked in $\mathcal{X}^{\dagger}$. Then a fibrant replacement in the model structure for Cartesian fibrations over $\mathcal{C}$ gives a model for $\mathrm{L}_{W}^{\mathrm{e}}\left(\pi^{\dagger}\right)$.

Lemma 2 Let $\mathcal{E}^{\dagger}$ be a marked $\infty$-category together with a functor $p: \mathcal{E} \rightarrow \mathcal{C}$. Consider $\mathfrak{F}(p)^{\dagger}: \mathfrak{F}(\mathcal{E})^{\dagger} \rightarrow \mathcal{C}$ as in Definition 5. Then for every vertex $c \in \mathcal{C}$ there is an equivalence of $\infty$-categories

$$
\mathrm{L}_{W}^{\mathcal{e}}\left(\mathfrak{F}(p)^{\dagger}\right) \times e\{c\} \simeq \mathrm{L}_{W}\left(\mathcal{E}_{c /}^{\dagger}\right)
$$

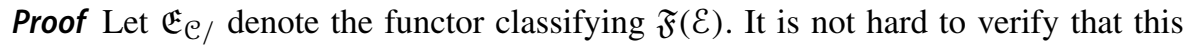
functor maps each $c \in \mathcal{C}$ to the $\infty$-category $\mathcal{E}_{c /}$ and that its action on morphisms is 
induced by precomposition in $\mathcal{C}$. Therefore we define $\widetilde{\mathfrak{E}}_{\mathcal{C}}^{\dagger}$, to be the functor sending each $c$ to $\mathcal{E}_{c /}^{\dagger}$. It is clear that we have an equivalence of Cartesian fibrations

$$
\mathrm{L}_{W}^{\mathcal{e}}\left(\mathfrak{F}(p)^{\dagger}\right) \simeq \mathrm{L}_{W}^{\mathcal{e}}\left(\operatorname{Un}_{\mathcal{C}}\left(\widetilde{\mathfrak{E}}_{\mathcal{C} / /}^{\dagger}\right)\right)
$$

In addition, we observe that the right-hand side can be modeled by a fibrant replacement of $\widetilde{\mathfrak{E}}_{\mathcal{C}}^{\dagger}$ in the projective model structure of $\mathrm{Set}_{\Delta}^{+}$-valued functors. This finally implies

$$
\mathrm{L}_{W}^{\mathcal{e}}\left(\mathrm{Un}_{\mathcal{C}}\left(\widetilde{\mathfrak{E}}_{\mathcal{C} /}^{\dagger}\right)\right) \times \mathcal{e}\{c\} \simeq \mathrm{L}_{W}\left(\mathcal{E}_{c /}^{\dagger}\right)
$$

\section{Weighted colimits in $\infty$-bicategories}

Definition 8 Let $F: \mathcal{C} \rightarrow \mathbb{B}$ and $W:$ eop $\rightarrow \mathfrak{C a t}_{\infty}$. We define a functor as the composite

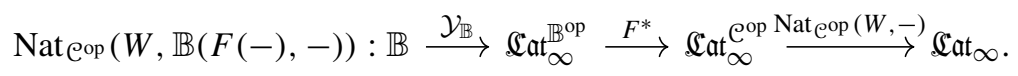

Definition 9 Let $F: \mathcal{C} \rightarrow \mathbb{B}$ and $W: \mathcal{C}^{o p} \rightarrow \mathfrak{L a t}_{\infty}$. We say that an object $b \in \mathbb{B}$ is the colimit of $F$ weighted by $W$ if there exists an equivalence of functors

$$
\mathbb{B}(b,-) \stackrel{\simeq}{\Longrightarrow} \operatorname{Nat}_{\odot o p}(W, \mathbb{B}(F(-),-))
$$

Remark 8 Since weighted colimits are unique up to equivalence we will often speak of "the weighted colimit" and denote it by $W \otimes F$.

Definition 10 Let $\mathcal{C}$ be an $\infty$-category. The twisted arrow $\infty$-category $\operatorname{Tw}(\mathcal{C})$ of $\mathcal{C}$ is the simplicial set given by $\operatorname{Hom}_{\mathrm{Set}_{\Delta}}\left(\Delta^{n}, \operatorname{Tw}(\mathcal{C})\right) \simeq \operatorname{Hom}_{\mathrm{Set}_{\Delta}}\left(\Delta^{n} \star\left(\Delta^{n}\right)^{\mathrm{op}}, \mathcal{C}\right)$. Note that $\operatorname{Tw}(\mathcal{C})$ comes equipped with a projection functor $\operatorname{Tw}(\mathcal{C}) \rightarrow \mathcal{C} \times \mathrm{C}^{\text {op }}$ which is a right fibration by Proposition 5.2.1.3 in [11], so that $\mathrm{Tw}(\mathcal{C})$ is an $\infty$-category.

We present now two of the main results obtained in [2] that will allow us to understand weighted colimits in $\mathfrak{l}_{\mathfrak{a t}}$. The proofs can be found in the aforementioned document as Theorem 4.3 and $* * *$ Theorem 4.28.

Proposition 2 Let $\mathcal{C}$ be $a \infty$-category and $\mathbb{D}$ an $\infty$-bicategory. Then for every pair of functors $F, G: \mathcal{C} \rightarrow \mathbb{D}$ there exists a equivalence of $\infty$-categories

$$
\operatorname{Nat}_{\mathcal{C}}(F, G) \rightarrow \lim _{\operatorname{Tw}(\mathcal{C})^{\text {op }}} \operatorname{Map}_{\mathbb{D}}(F(-), G(-))
$$

which is natural in each variable. 
Corollary 2 Let $F: \mathcal{C} \rightarrow \mathfrak{C a t}_{\infty}$ and $W: \mathrm{e}^{\mathrm{op}} \rightarrow \mathfrak{C} \mathfrak{a t}_{\infty}$. Then there is an equivalence of $\infty$-categories

$$
W \otimes F \stackrel{\simeq}{\longrightarrow} \operatorname{colim}_{\mathrm{Tw}(\mathcal{C})} W \times F
$$

We finish the section by relating conical weighted colimits, i.e. those with weight constant on the terminal category, with ordinary colimits.

Proposition 3 Let $F: \mathcal{C} \rightarrow \mathbb{B}$ and let $\underline{*}: \mathrm{Cop}^{\rightarrow} \mathfrak{C a t}_{\infty}$ denote the constant functor with value the terminal $\infty$-category. Suppose that $\underset{*}{\otimes} F$ exists. Then $\operatorname{colim}_{\mathcal{C}} F$ exists and there is an equivalence in $\mathcal{B}$

$$
\underline{*} \otimes F \stackrel{\simeq}{\rightarrow} \operatorname{colim}_{\mathcal{C}} F
$$

where the right-hand side is given by the $\infty$-categorical colimit of $F$ in the underlying $\infty$-category $\mathcal{B} \subseteq \mathbb{B}$.

Proof Let $\Xi: \mathbb{B}(\underline{*} \otimes F,-) \stackrel{\simeq}{\rightarrow} \operatorname{Nat} \operatorname{Cop}(\underline{*}, \mathbb{B}(F(-),-))$ be the natural equivalence exhibiting $\underline{*} \otimes F$ as the weighted colimit of $F$ and let $\iota^{*} \Xi$ denote its restriction to $\mathcal{B}$. Observe that $\iota^{*} \Xi$ factors through $\mathcal{C}_{\infty}$. We will abuse notation by viewing $\iota^{*} \Xi$ as a functor with target $\mathrm{Cat}_{\infty}$. Now we consider the following composite

$$
\mathcal{B} \times \Delta^{1} \stackrel{\iota^{*} \Xi}{\longrightarrow} \operatorname{Cat}_{\infty} \stackrel{k}{\rightarrow} \mathcal{S}
$$

where $k$ denotes the underlying Kan complex functor. It is clear that we have produced a natural equivalence $\mathcal{B}(\underline{*} \otimes F,-) \stackrel{\simeq}{\rightarrow} \operatorname{Nat}_{\mathcal{C o p}}(\underline{*}, \mathcal{B}(F(-),-))$. Observe that due to Proposition 2 we have the following natural equivalence of spaces for every $b \in \mathcal{B}$

$$
\begin{aligned}
\operatorname{Nat}_{\mathcal{C o p}}(\underline{*}, \mathcal{B}(F(-), b)) & \simeq \lim _{\operatorname{Tw}(\mathcal{C}) \text { op }} \mathcal{S}(*, \mathcal{B}(F(-), b)) \simeq \lim _{\operatorname{Tw}(\mathcal{C})^{\text {op }}} \mathcal{B}(F(-), b) \\
\lim _{\operatorname{Tw}(\mathcal{C})^{\text {op }}} \mathcal{B}(F(-), b) & \simeq \operatorname{Nat}_{\mathcal{C}}(F, \underline{b})
\end{aligned}
$$

where $\underline{b}$ denotes the constant functor with value $b \in \mathcal{B}$. We have thus produced equivalences of functors

$$
\mathcal{B}(\underline{*} \otimes F,-) \stackrel{\simeq}{\rightarrow} \operatorname{Nat}_{\mathcal{C o p}}(\underline{*}, \mathcal{B}(F(-),-)) \stackrel{\simeq}{\rightarrow} \operatorname{Nat}_{\mathcal{C}}(F, \underline{(-)})
$$

We note that $\mathcal{B}_{/ \notin \otimes F}$ is the left fibration classifying the functor $\mathcal{B}(\underline{*} \otimes F,-)$ and that it has an initial object. Therefore the left fibration classifying Nate $(F,(-))$ has an initial object. This exhibits $\underline{*} \otimes F$ as the colimit of $F: \mathcal{C} \rightarrow \mathcal{B}$. 


\section{Marked colimits}

\subsection{Definitions and general properties}

Definition 11 Let $\mathcal{C}^{\dagger}$ be a marked $\infty$-category and consider $\mathfrak{F}\left(\operatorname{id}_{\mathcal{C}}\right): \mathfrak{F}(\mathcal{C})^{\dagger} \rightarrow \mathcal{C}$ as in Definition 5. Given the Cartesian fibration $\mathrm{L}_{W}^{\mathcal{C}}\left(\mathfrak{F}\left(\operatorname{id}_{\mathcal{C}}\right)^{\dagger}\right)$ discussed in Definition 7 we let

$$
\mathfrak{C}_{\mathcal{C} /}^{\dagger}: \mathfrak{C}^{\text {op }} \rightarrow \mathfrak{C a t}_{\infty}
$$

be its associated functor. As we noted in Lemma 2, the functor $\mathfrak{C}_{\mathcal{C} /}^{\dagger}$ sends each $c \in \mathcal{C}$ to the localized comma category $\mathrm{L}_{W}\left(\mathrm{C}_{c /}^{\dagger}\right)$

Definition 12 Given a marked $\infty$-category $\mathcal{C}^{\dagger}$ and a diagram $F: \mathcal{C} \rightarrow \mathbb{B}$ we define

$$
\operatorname{colim}^{\dagger} \text { e } F:=\mathfrak{C}_{\mathcal{C} /}^{\dagger} \otimes F
$$

and call it the marked colimit of $F$.

Remark 9 Using Corollary 2 we see that if $\mathbb{B}=\mathfrak{C a t}_{\infty}$ and the marking of $\mathcal{C}$ consists only of equivalences our definition coincides with the definition of lax colimit given in [6].

Theorem 6 Let $F: \mathcal{C} \rightarrow \mathbb{B}$ and suppose that colim $^{]}{ }_{\mathrm{C}} F$ exists. Then the $\infty$-categorical colimit of $F$ in the underlying $\infty$-category $\mathcal{B} \subseteq \mathbb{B}$ exists and there is an equivalence

$$
\operatorname{colim}^{\dagger} \mathrm{e} F \stackrel{\simeq}{\rightarrow} \operatorname{colim}_{\mathcal{C}} F
$$

Proof Let $t: \mathfrak{C}_{\mathrm{C} /}^{\sharp} \Rightarrow \underline{*}$ denote the unique map to the terminal functor. By Proposition 3 it will suffice to show that $t$ is a levelwise equivalence. This follows immediately from Lemma 2 and the fact that slice categories $\mathcal{C}_{c}$ are all contractible.

Remark 10 It is natural to ask ourselves, whether Theorem 6 admits a converse. The answer to this question is no and it is already well documented (see for example 3.54 in [8]). For the sake of completeness we will include an example here. Let $\mathbb{B}$ be the following 2-category

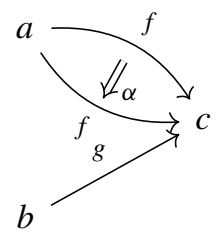

Then it is clear that $c$ is the coproduct of $a$ and $b$ in the underlying 1-category $\mathcal{B}$. However $c$ fails to satisfy the 2-dimensional universal property because the identity morphism on $c$ has no non-trivial 2-morphisms. 
Corollary 3 Let $\mathrm{C}^{\dagger}$ be a marked $\infty$-category and consider a functor $F: \mathcal{C}^{\dagger} \rightarrow \mathcal{B}$ with $\mathcal{B}$ an $\infty$-category. Then colim ${ }^{\dagger} \mathrm{e} F$ exists if and only if the colimit of $F$ exists and there is an equivalence in $\mathcal{B}$

$$
\operatorname{colim}^{\dagger} \mathrm{e} F \stackrel{\simeq}{\rightarrow} \operatorname{colim}_{C} F
$$

Proof It follows from Theorem 6 after noting that since the mapping categories in $\mathcal{B}$ are Kan complexes then any natural transformation $\mathfrak{C}_{\mathcal{C} /}^{\dagger} \Rightarrow \mathcal{B}(F(-), b)$ must factor

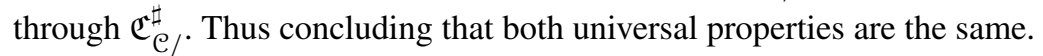

Proposition 4 Let $\pi: X \rightarrow$ C be a Cartesian fibration where we view $\mathcal{X}$ as a marked category (denoted by $X^{\natural}$ ) by marking the Cartesian edges. Consider the base change adjunction

$$
\pi !: \mathrm{Cat}_{\infty / X}^{\mathrm{cart}} \rightleftarrows: \mathrm{Cat}_{\infty / \mathcal{C}}^{\mathrm{cart}}: \pi^{*}
$$

Then for every Cartesian fibration $y \rightarrow \mathcal{C}$ there is an equivalence of $\infty$-categories

$$
\operatorname{Fun}_{X}^{c a r t}\left(\operatorname{L}_{W}^{x}\left(\mathfrak{F}(\operatorname{id} x)^{\natural}\right), \pi^{*} y\right) \rightarrow[\simeq] \operatorname{Fun}_{\mathcal{C}}^{\operatorname{cart}}(X, y)
$$

natural in $y$. In particular, there is an equivalence of Cartesian fibrations $\pi ! \mathrm{L}_{W}$ $\left(\mathfrak{F}(\operatorname{id} x)^{\natural}\right) \simeq X$.

Proof We can produce the following natural equivalences

$$
\operatorname{Fun}_{X}^{\operatorname{cart}}\left(\operatorname{L}_{W}^{X}\left(\mathfrak{F}\left(\operatorname{id}_{X}\right)^{\natural}\right), \pi^{*} y\right) \simeq \operatorname{Fun}_{X}^{\natural}\left(X, \mathfrak{U}\left(\pi^{*} y\right)\right) \simeq \operatorname{Fun}_{\mathcal{C}}^{\natural}(X, \mathfrak{U}(y))=\operatorname{Fun}_{\mathcal{C}}^{\text {cart }}(X, y)
$$

where the third equivalence is given by the non-Cartesian base change adjunction. The result follows.

Theorem 7 Let $F: \mathcal{C} \rightarrow \mathbb{B}$ and $W: e^{o p} \rightarrow \mathfrak{C a t}_{\infty}$. Let $p: \mathcal{W} \rightarrow \mathcal{C}$ denote the Cartesian fibration classifying $W$. Suppose that $W \otimes F$ exists. Then $\operatorname{colim}_{\mathcal{W}}^{\natural}$ exists and there is an equivalence in $\mathbb{B}$

$$
W \otimes F \stackrel{\simeq}{\longrightarrow} \operatorname{colim}_{\mathcal{W}}^{\natural} F \circ p
$$

Proof Let $\mathfrak{W}_{\mathcal{W} \text { / }}^{\natural}$ denote the weight functor in the definition of the colimit of $F \circ p$. By Proposition 4 we know that $p_{!} \mathfrak{W}_{\mathcal{W} /}^{\natural} \simeq W$. This shows that there is a map

$$
\operatorname{Nat}_{\mathcal{C o p}}(W, \mathbb{B}(F(-),-)) \Rightarrow \operatorname{Nat}_{\mathcal{W}} \text { op }\left(\mathfrak{W}_{\mathcal{W} /}^{\natural}, \mathbb{B}((F \circ p)(-),-)\right)
$$


which is levelwise an equivalence on the underlying Kan complexes. Combining Proposition 2 and Proposition 6.9 in [6] we obtain for every $b \in \mathbb{B}$ the following commutative diagram

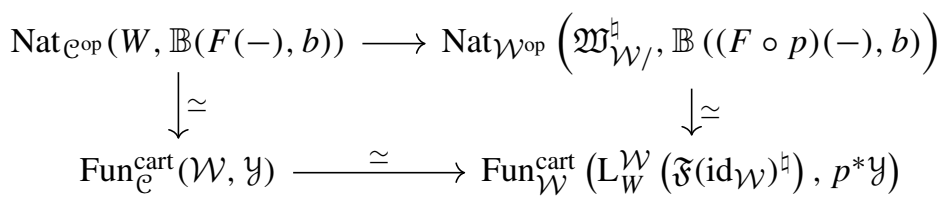

where $y$ is the Cartesian fibration classifying $\mathbb{B}(F(-), b)$. Since the bottom horizontal map is an equivalence due to Proposition 4 we conclude by 2-out-of-3.

\subsection{Marked colimits in the $\infty$-bicategory of $\infty$-categories.}

In this section we show how to compute marked colimits of functors with values in $\infty$ categories. Our strategy will be a direct generalization of the arguments presented in [6] where the authors show that the unstraightening functor (see Notation 7) computes the lax colimit of a functor.

Definition 13 Let $F: \mathcal{C} \rightarrow \mathcal{C a t}_{\infty}$ be a functor and denote by $\mathcal{F} \rightarrow \mathcal{C}$ its associated coCartesian fibration. Given $X \in \mathcal{C} a_{\infty}$ we define a simplicial set $\Phi_{X}^{\mathcal{F}}$ over $\mathcal{C}$ via the universal property $\operatorname{Hom}_{\mathcal{C}}\left(K, \Phi_{\mathcal{X}}^{\mathcal{F}}\right) \simeq \operatorname{Hom}\left(K \times \times_{\mathcal{C}} \mathcal{F}, \mathcal{X}\right)$.

Remark 11 As a special case of (the dual of) Corollary 3.2.2.12 in [10] we see that $\Phi_{X}^{\mathcal{F}} \rightarrow \mathcal{C}$ is a Cartesian fibration. An edge $\Delta^{1} \rightarrow \Phi_{X}^{\mathcal{F}}$ is Cartesian if and only if the associated functor $\Delta^{1} \times \mathcal{e} \mathcal{F} \rightarrow X$ maps coCartesian edges in $\Delta^{1} \times \mathfrak{e} \mathcal{F}$ to equivalences in $X$.

Proposition 5 The Cartesian fibration $\Phi_{X}^{\mathcal{F}} \rightarrow \mathcal{C}$ classifies the functor

$$
\operatorname{Fun}(F(-), X): \mathcal{C}^{\mathrm{op}} \rightarrow \mathrm{Cat}_{\infty}
$$

Proof This is Proposition 7.3 of [6].

Definition 14 Let $\mathrm{C}^{\dagger}$ be a marked $\infty$-category and consider a Cartesian (resp. coCartesian) fibration $X \rightarrow \mathcal{C}$. We equip $X$ with a marking by declaring an edge marked if and only if it is Cartesian (resp. coCartesian) and its image in $\mathcal{C}$ is marked. We will denote this marked $\infty$-category over $\mathcal{C}$ by $X^{\natural(\dagger)}$.

Remark 12 Let $\mathcal{C}^{\dagger}$ be a marked $\infty$-category and consider a functor $F: \mathcal{C} \rightarrow \mathcal{C a t}_{\infty}$. Denote its associated coCartesian fibration by $\mathcal{F}$. Then given $\mathcal{X} \in \mathcal{C a t}_{\infty}$ we have a natural equivalence of $\infty$-categories

$$
\operatorname{Fun}\left(\mathrm{L}_{W}\left(\mathcal{F}^{\natural(\dagger)}\right), X\right) \simeq \operatorname{Fun}^{\natural(\dagger)}(\mathcal{F}, X) \simeq \operatorname{Fun}_{\mathcal{C}}^{\dagger}\left(\mathcal{C}, \Phi_{X}^{\mathcal{F}}\right)
$$

where the first equivalence is the universal property of the localization (see Notation 8 ) and the second is given by the universal property of $\Phi_{X}^{\mathcal{F}}$. 
Proposition 6 Let $\mathcal{C}$ be an $\infty$-category. Given $F, G: \mathrm{C}^{\mathrm{op}} \rightarrow \mathrm{Cat}_{\infty}$ classified by the Cartesian fibrations $\mathcal{F}$ and $\mathcal{G}$ respectively, there is a natural equivalence of $\infty$ categories

$$
\operatorname{Fun}_{\mathcal{C}}^{\text {cart }}(\mathcal{F}, \mathcal{G}) \simeq \lim _{\operatorname{Tw}(\mathcal{C})^{\text {op }}} \operatorname{Fun}(F(-), G(-))
$$

Proof See Proposition 6.9 in [6].

Theorem 8 Let $\mathrm{C}^{\dagger}$ be a marked $\infty$-category. Given $F: \mathcal{C} \rightarrow \mathfrak{C a t}_{\infty}$ there is a equivalence of $\infty$-categories

$$
\mathrm{L}_{W}\left(\mathrm{Un}_{\mathcal{C}}^{\mathrm{co}}(F)^{\natural(\dagger)}\right) \simeq \operatorname{colim}^{\dagger} \mathrm{e} F
$$

Proof We fix the notation $\operatorname{Un}_{\mathcal{C}}^{c o}(F)^{\natural(\dagger)}=\mathcal{F}^{\natural}(\dagger)$. We have a natural equivalence of $\infty$-categories provided by Lemmas 1 and 12

$$
\operatorname{Fun}\left(\mathrm{L}_{W}\left(\mathcal{F}^{\natural(\dagger)}\right), X\right) \simeq \operatorname{Fun}_{\mathcal{C}}^{\dagger}\left(\mathcal{C}, \Phi_{X}^{\mathcal{F}}\right) \simeq \operatorname{Fun}_{\mathcal{C}}^{\text {cart, } \dagger}\left(\mathfrak{F}(\mathcal{C}), \Phi_{X}^{\mathcal{F}}\right) \simeq \operatorname{Fun}_{\mathcal{C}}^{\text {cart }}\left(\mathrm{L}_{W}^{\mathcal{C}}\left(\mathfrak{F}\left(\operatorname{id}_{\mathcal{C}}\right)^{\dagger}\right), \Phi_{X}^{\mathfrak{F}}\right)
$$

Propositions 5 and 6 in turn imply

$\operatorname{Fun}_{\mathcal{C}}^{\text {cart }}\left(\mathrm{L}_{W}^{\mathcal{C}}\left(\mathfrak{F}\left(\operatorname{id}_{\mathcal{C}}\right)^{\dagger}\right), \Phi_{X}^{\mathfrak{F}}\right) \simeq \lim _{\operatorname{Tw}(\mathcal{C})^{\text {op }}} \operatorname{Fun}\left(\mathfrak{C}_{\mathcal{C} /}^{\dagger}, \operatorname{Fun}(F(-), X)\right) \simeq \operatorname{Fun}\left(\operatorname{colim}_{\mathrm{Tw}(\mathcal{C})} \mathfrak{C}_{\mathcal{C} /}^{\dagger} \times F, X\right)$

Combining these two natural equivalences, the result follows from the Yoneda lemma and Corollary 2.

Corollary 4 Let $\mathrm{C}$ be an $\infty$-category. Given $F: \mathcal{C} \rightarrow \mathfrak{L a t}_{\infty}$ there is an equivalence of $\infty$-categories

$$
\mathrm{Un}_{\mathcal{C}}^{\mathrm{co}}(F) \simeq \operatorname{colim}_{\mathcal{C}}^{b} F
$$

Proof This follows by observing that a coCartesian edge lying over a degenerate edge must be an equivalence.

As a consequence of Theorem 8, we obtain an alternative proof of Corollary 3.3.4.3 in [10].

Corollary 5 Let $F: \mathcal{C} \rightarrow \mathcal{C a t}_{\infty}$ then there is an equivalence of $\infty$-categories

$$
\mathrm{L}_{W}\left(\mathrm{Un}_{\mathcal{C}}^{\mathrm{co}}(F)^{\natural}\right) \simeq \operatorname{colim}_{\mathcal{C}} F
$$

Proof Combine Theorem 8 with $\mathcal{C}^{\sharp}$ and Proposition 3 . 


\section{A cofinality criterion}

The goal of this section is to extend the preexisting theory of cofinality to the setting of marked colimits. Our main result, Theorem 9, is a generalization of the characterization of cofinal functors appearing in Theorem 4.1.3.1 in [10]. As an immediate corollary we obtain a generalization of Quillen's theorem A for marked $\infty$-categories.

Definition 15 Let $f: \mathcal{C}^{\dagger} \rightarrow \mathcal{D}^{\dagger}$ be a marked functor and consider $\mathfrak{F}(f): \mathfrak{F}(\mathcal{C})^{\dagger} \rightarrow \mathcal{D}$ as in Definition 5 . We denote by

$$
\mathfrak{C}_{\mathcal{D} /}^{\dagger}: \mathcal{D}^{\text {op }} \rightarrow \mathfrak{l a t}_{\infty}
$$

the functor classifying the Cartesian fibration $\mathrm{L}_{W}^{\mathcal{D}}\left(\mathfrak{F}(f)^{\dagger}\right)$.

Remark 13 Observe that we have a natural transformation $\mathcal{A}_{f}: \mathfrak{C}_{\mathcal{D} /}^{\dagger} \Rightarrow \mathfrak{D}_{\mathcal{D} / \text {. We will }}^{\dagger}$ abuse notation by also denoting by $\mathcal{A}_{f}$ the associated map of Cartesian fibrations.

Proposition 7 Let $f: \mathrm{C}^{\dagger} \rightarrow \mathcal{D}^{\dagger}$ be a marked functor and let $f ! \dashv f^{*}$ denote the base change adjunction

$$
f_{!}: \mathrm{Cat}_{\infty / \mathcal{C}}^{\mathrm{cart}} \rightleftarrows: \mathrm{Cat}_{\infty / \mathcal{D}}^{\mathrm{cart}}: f^{*}
$$

Then there is a natural equivalence of $\infty$-categories

$$
\operatorname{Fun}_{\mathcal{C}}^{\text {cart }}\left(\mathrm{L}_{W}^{\mathcal{e}}\left(\mathfrak{F}\left(\operatorname{id}_{\mathcal{C}}\right)^{\dagger}\right), f^{*} x\right) \stackrel{\simeq}{\rightarrow} \operatorname{Fun}_{\mathcal{D}}^{\text {cart }}\left(\mathrm{L}_{W}^{\mathcal{D}}\left(\mathfrak{F}(f)^{\dagger}\right), x\right)
$$

In particular, we have $f_{!} \mathrm{L}_{W}^{\mathcal{C}}\left(\mathfrak{F}(\mathrm{id} \mathcal{C})^{\dagger}\right) \stackrel{\simeq}{\rightarrow} \mathrm{L}_{W}^{\mathcal{D}}\left(\mathfrak{F}(f)^{\dagger}\right)$.

Proof Let $X \rightarrow \mathcal{D}$ be a Cartesian fibration. We observe that we have natural equivalences

$$
\operatorname{Fun}_{\mathcal{C}}^{\text {cart }}\left(\mathrm{L}_{W}^{\mathcal{C}}\left(\mathfrak{F}\left(\operatorname{id}_{\mathcal{C}}\right)^{\dagger}\right), f^{*} X\right) \simeq \operatorname{Fun}_{\mathcal{C}}^{\dagger}\left(\mathcal{C}, \mathfrak{U}\left(f^{*} X\right)\right) \simeq \operatorname{Fun}_{\mathcal{D}}^{\dagger}(\mathcal{C}, \mathfrak{U}(X))
$$

where the second equivalence is given by the non-Cartesian base change adjunction $f_{*} \dashv f^{*}$. It is clear that the right-hand side is equivalent to Fun $\operatorname{Part}_{\mathcal{D}}^{\text {cart }}\left(\mathrm{L}_{W}^{\mathcal{D}}\left(\mathfrak{F}(f)^{\dagger}\right), X\right)$ and the result follows.

Given a marked functor $f: \mathcal{C}^{\dagger} \rightarrow \mathcal{D}^{\dagger}$ it follows that for every functor $G: \mathcal{D}^{\text {op }} \rightarrow$ $\mathfrak{L a t}_{\infty}$ we can produce the following natural transformations

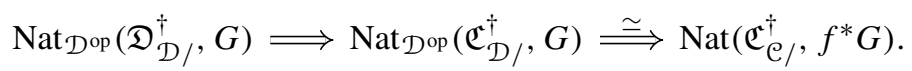

where the last map is a natural equivalence by virtue of Proposition 7. It follows that when $G=\mathbb{B}(F(-), b)$ for some diagram $F: \mathcal{D} \rightarrow \mathbb{B}$, we obtain a map

$$
\operatorname{colim}^{\dagger} \mathrm{e} F \circ f \rightarrow \operatorname{colim}^{\dagger} \mathcal{D} F
$$


which we will call the canonical comparison map whenever both are defined.

Definition 16 A functor $f: \mathcal{C}^{\dagger} \rightarrow \mathcal{D}^{\dagger}$ of marked $\infty$-categories is said to be marked cofinal if for every functor $F: \mathcal{D} \rightarrow \mathbb{B}$, the following conditions hold:

- The marked colimit $F$ exists if and only the marked colimit of $(F \circ f)$ exists.

- The canonical comparison map $\operatorname{colim}^{\dagger} \mathrm{e} F \circ f \stackrel{\simeq}{\longrightarrow} \operatorname{colim}^{\dagger} \mathcal{D} F$ is an equivalence in $\mathbb{B}$.

Proposition 8 Let $f: \mathcal{C}^{\dagger} \rightarrow \mathcal{D}^{\dagger}$ be a marked functor. For every diagram $F: \mathcal{D} \rightarrow$ $\mathfrak{L a t}_{\infty}$ we have a commutative diagram in $\mathrm{Cat}_{\infty}$ given by

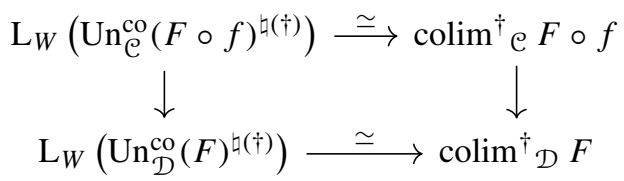

where the leftmost vertical map is induced by pullback along $f$.

Proof This follows as a corollary of Theorem 8.

Proposition 9 A functor $f: \mathcal{C}^{\dagger} \rightarrow \mathcal{D}^{\dagger}$ of marked $\infty$-categories is marked cofinal if and only if the natural transformation

$$
\mathcal{A}_{f}: \mathfrak{C}_{\mathcal{D} /}^{\dagger} \Rightarrow \mathfrak{D}_{\mathcal{D} /}^{\dagger}
$$

is a levelwise equivalence.

Proof Suppose $f$ is marked cofinal. Given an object $d \in \mathcal{D}$ we define the composite

$$
\mathcal{Y}_{d}: \mathcal{D} \rightarrow \mathcal{S} \subseteq \mathrm{Cat}_{\infty}
$$

where first functor is given by $\operatorname{Map}_{\mathcal{D}}(d,-)$ and the second functor is the inclusion of the full subcategory of spaces. Then using Corollary 8 we obtain an equivalence of $\infty$-categories

$$
\mathrm{L}_{W}\left(\mathcal{C}_{d /}^{\dagger}\right) \stackrel{\simeq}{\longrightarrow} \mathrm{L}_{W}\left(\mathcal{D}_{d /}^{\dagger}\right)
$$

Using Lemma 2 we identify this map with the fiber of the map $\mathcal{A}_{f}$ over $d$. Since equivalences of Cartesian fibrations can be detected fiberwise it follows that $\mathcal{A}_{f}$ is an equivalence. The converse follows immediately.

Remark 14 Suppose $f: \mathcal{C}^{\sharp} \rightarrow \mathcal{D}^{\sharp}$ is cofinal for the maximal marking. Since $f$ is cofinal we obtain an equivalence after $\infty$-groupoid completion

$$
\mathrm{L}_{W}\left(\mathcal{C}_{d /}^{\sharp}\right) \simeq \mathrm{L}_{W}\left(\mathcal{D}_{d /}^{\sharp}\right)
$$

Then it follows that $f$ satisfies the hypothesis of Theorem 4.1.3.1 in [10] and so pullback along $f$ preserves all $\infty$-limits. 
Corollary 6 (Theorem $\mathrm{A}^{\dagger}$ ) Let $f: \mathrm{C}^{\dagger} \rightarrow \mathcal{D}^{\dagger}$ be a marked cofinal functor. Then there exists an equivalence of $\infty$-categories

$$
\mathrm{L}_{W}\left(\mathrm{C}^{\dagger}\right) \stackrel{\simeq}{\rightarrow} \mathrm{L}_{W}\left(\mathcal{D}^{\dagger}\right)
$$

Proof By Theorem 8 we can identify $\mathrm{L}_{W}\left(\mathcal{D}^{\dagger}\right)$ with the marked colimit of the constant point valued functor. The result then follows from Corollary 8.

Remark 15 For the rest of this section, we will abuse notation by denoting the Cartesian fibration $\mathrm{L}_{W}^{\mathcal{D}}\left(\mathfrak{F}(f)^{\dagger}\right)$ by $\mathfrak{C}_{\mathcal{D}}^{\dagger}$ and similarly for the other fiberwise localizations of free fibrations already mentioned.

Lemma 3 Let $\mathcal{D}^{\dagger}$ be a marked $\infty$-category and consider the Cartesian fibration $\mathfrak{D}_{\mathcal{D} /}^{\dagger} \rightarrow \mathcal{D}$. Then the following hold

- For every $d \in \mathcal{D}$ there exists an initial object in $\mathfrak{D}_{\mathcal{D} /}^{\dagger} \times_{\mathcal{D}}\{d\}$.

- Every object in $\mathfrak{D}_{\mathcal{D} /}^{\dagger} \times \mathcal{D}\{d\}$ represented by a marked morphism is initial.

Proof The proof is analogous to the proof of Lemma 4.0.3 in [1].

Proposition 10 Let $\pi: X \rightarrow \mathcal{D}$ be a Cartesian fibration of simplicial sets. Assume that for each vertex $c \in \mathcal{C}$, the $\infty$-category $X_{d}$ has an initial object. Denote by $X^{\prime} \subset X$ the full simplicial subset of $\mathcal{X}$ spanned by those $x$ which are initial objects in $X_{\pi(x)}$. Then

$$
\pi \mid X^{\prime}: X^{\prime} \rightarrow \mathcal{C}
$$

is a trivial Kan fibration of simplicial sets. Moreover, a section s of $\pi: \mathcal{X} \rightarrow \mathcal{C}$ is initial in the $\infty$-category $\operatorname{Fun}_{\mathcal{C}}(\mathcal{C}, X)$ if and only if $s$ factors through $X^{\prime}$.

Proof See Proposition 2.4.4.9 in [10].

Theorem 9 Let $f: \mathcal{C}^{\dagger} \rightarrow \mathcal{D}^{\dagger}$ be a functor of marked $\infty$-categories. Then $f$ is marked cofinal if and only the following conditions hold

1. For every $d \in \mathcal{D}$ there is a morphism $g_{d}: d \rightarrow f(c)$ which is initial in $\mathrm{L}_{W}\left(\mathrm{C}_{d /}^{\dagger}\right)$ and in $\mathrm{L}_{W}\left(\mathcal{D}_{d /}^{\dagger}\right)$.

2. Every object in $\mathrm{L}_{W}\left(\mathrm{e}_{d /}^{\dagger}\right)$ represented by a marked morphism in $\mathcal{D}^{\dagger}$ is initial.

3. There exists a Cartesian morphism providing a solution to the lifting problem

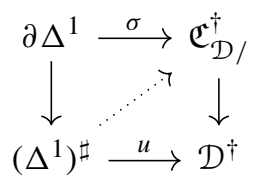

if the image of $\sigma$ consists in two initial objects in their respective fibers. 
Proof By Proposition 9 it will suffice to show that $\mathcal{A}_{f}$ is an equivalence of Cartesian fibrations precisely when the conditions above are satisfied.

Suppose that $\mathcal{A}_{f}$ is an equivalence and pick an inverse $\Xi: \mathfrak{D}_{\mathcal{D} /}^{\dagger} \rightarrow \mathfrak{C}_{\mathcal{D} /}^{\dagger}$ over $\mathcal{D}$. First we see that the first 2 conditions can be checked by noting that $\mathcal{A}_{f}$ induces an equivalence of $\infty$-categories upong passage to fibers. The existence of $\Xi$ implies the existence of a section $s_{f}: \mathcal{D} \rightarrow \mathfrak{C}_{\mathcal{D}}^{\dagger}$, mapping each object of $\mathcal{D}$ to an initial object in the fiber and mapping marked edges to Cartesian edges. To finish the proof of this implication we will show that condition (3) holds. We start by picking a Cartesian lift of $u$ denoted by $\hat{u}$ such that $\hat{u}(1)=\sigma(1)$. Then since $\sigma(0)$ is initial we obtain a morphism from $\sigma(0) \rightarrow \hat{u}(0)$. Note that if we show that $\hat{u}(0)$ is initial its corresponding fibre then we can finish our argument by picking an adequate composite $\sigma(0) \rightarrow \hat{u}(0) \rightarrow$ $\hat{u}(1)=\sigma(1)$. Since $\mathcal{A}_{f}$ is an equivalence it will suffice that $\mathcal{A}_{f}(\hat{u}(0))=y$ is initial. We note that $y$ is equivalent to the morphism $u$ viewed as an object of $\mathfrak{D}_{\mathcal{D}}^{\dagger}$, therefore the conclusion follows from Lemma 3.

To show the converse we first observe that condition (1) together with Proposition 10 imply the existence of a section $s_{f}: \mathcal{D} \rightarrow \mathfrak{C}_{\mathcal{D}}^{\dagger}$ such that for every $d \in \mathcal{D}$ both $s_{f}(d)$ and $\mathcal{A}_{f}\left(s_{f}(d)\right)$ are initial in their respective fibers. We claim that $s_{f}$ maps marked edges to Cartesian edges in $\mathfrak{C}_{\mathcal{D} /}^{\dagger}$. To see this given $\alpha:\left(\Delta^{1}\right)^{\sharp} \rightarrow \mathcal{D}^{\dagger}$ we denote by $\widetilde{\alpha}$ the Cartesian lift provided by condition (3). One immediately checks that there exists an equivalence in $\Delta^{1} \stackrel{u}{\rightarrow} \mathfrak{C}_{\mathcal{D}}^{\dagger}$, such that $s_{f}(\alpha) \sim u \circ \widetilde{\alpha}$ and thus $s_{f}(\alpha)$ is Cartesian. In particular we obtain a map of Cartesian fibrations

$$
\Xi: \mathfrak{D}_{\mathcal{D} /}^{\dagger} \rightarrow \mathfrak{C}_{\mathcal{D} /}^{\dagger}
$$

We fix the notation

$$
\Gamma_{\mathcal{D}}: \operatorname{Fun}_{\mathcal{D}}^{\text {cart }}\left(\mathfrak{D}_{\mathcal{D} /}^{\dagger}, \mathfrak{D}_{\mathcal{D} /}^{\dagger}\right) \stackrel{\simeq}{\rightarrow} \operatorname{Fun}_{\mathcal{D}}^{\dagger}\left(\mathcal{D}, \mathfrak{D}_{\mathcal{D} /}^{\dagger}\right)
$$

and observe that both the identity functor on $\mathfrak{D}_{\mathcal{D} /}^{\dagger}$ and $\mathcal{A}_{f} \circ \Xi$ get mapped under $\Gamma_{\mathcal{D}}$ to sections landing in initial objects in the fibers. It follows from Proposition 10 that $\mathcal{A}_{f} \circ \Xi \sim i d$.

Similarly we consider

$$
\Gamma_{\mathcal{C}}: \operatorname{Fun}_{\mathcal{D}}^{\text {cart }}\left(\mathfrak{C}_{\mathcal{D} /}^{\dagger}, \mathfrak{C}_{\mathcal{D} /}^{\dagger}\right) \stackrel{\simeq}{\rightarrow} \operatorname{Fun}_{\mathcal{D}}^{\dagger}\left(\mathcal{C}, \mathfrak{C}_{\mathcal{D} /}^{\dagger}\right)
$$

and observe that $\Gamma_{\mathcal{C}}\left(\Xi \circ \mathcal{A}_{f}\right)(c)=s_{f}(f(c))$ which is initial in the fiber by construction. Similarly the image of the identity functor under $\Gamma_{\mathcal{C}}$ sends $c \in \mathcal{C}$ to an object represented by a marked morphism. Condition (2) implies that this object is initial. Since both maps can factored through the pullback

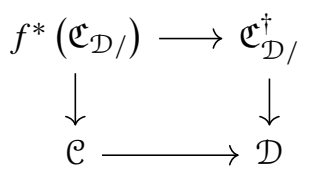


we can apply Proposition 10 to their factorizations. This shows that $\Xi \circ \mathcal{A}_{f} \sim$ id and thus finishes the proof.

Remark 16 Let $\mathfrak{C}_{\mathcal{D} /}^{\dagger}: \mathcal{D}^{\text {op }} \rightarrow \mathcal{C} t_{\infty}$. Note that condition (3) in Theorem 9 holds if and only if for every marked edge $d \stackrel{u}{\rightarrow} d^{\prime}$ in $\mathcal{D}$ the functor $\mathfrak{C}_{\mathcal{D} /}^{\dagger}(u)$ preserves initial objects.

Acknowledgements I would like to thank Tobias Dyckerhoff for the guidance and support offered throughout this process. It is also a pleasure to thank Walker Stern for all the careful revisions of the draft and the many improvements suggested. I am grateful to an anonymous referee for the very careful reading which helped improve the article. The author acknowledges the support of the VolkswagenStiftung through the Lichtenberg Professorship Programme.

Author Contributions Not applicable

Funding Open Access funding enabled and organized by Projekt DEAL. The author acknowledges the support of the VolkswagenStiftung through the Lichtenberg Professorship Programme.

Availability of data and material Not applicable.

\section{Declarations}

\section{Conflicts of interest Not applicable.}

Code availability Not applicable.

Open Access This article is licensed under a Creative Commons Attribution 4.0 International License, which permits use, sharing, adaptation, distribution and reproduction in any medium or format, as long as you give appropriate credit to the original author(s) and the source, provide a link to the Creative Commons licence, and indicate if changes were made. The images or other third party material in this article are included in the article's Creative Commons licence, unless indicated otherwise in a credit line to the material. If material is not included in the article's Creative Commons licence and your intended use is not permitted by statutory regulation or exceeds the permitted use, you will need to obtain permission directly from the copyright holder. To view a copy of this licence, visit http://creativecommons.org/licenses/by/4.0/.

\section{References}

1. Abellán, G.F., Stern, W.: Theorem A for marked 2-categories. 2020 arXiv: 2002.12817

2. Abellán, G.F., Stern, W.: Enhanced twisted arrow categories. arXiv:2009.11969 (2020)

3. Cisinski, D.: Higher Categories and Homotopical Algebra. Cambridge University Press, Cambridge (2019)

4. Berman, J.: On lax colimits in $\infty$-categories. arXiv: 2006.10851 (2020)

5. Descotte, M.E., Dubuc, E.J., Szyld, M.: Sigma limits in 2-categories and flat pseudofunctors. Adv. Math. 718, 266-313 (2018). https://doi.org/10.1016/j.aim.2018.05.021

6. Gepner, D., Haugseng, R., Nikolaus, T.: Lax colimits and free fibrations in $\infty$-categories. Doc. Math. 22, 1225-1266 (2015)

7. Gray, J.: The existence and construction of Lax limits. Cahiers Topol. Géométr. Différ. Catégoriques Tome 21(3), 277-304 (1980)

8. Kelly, G.M.: Basic concepts of enriched category theory. Reprints Theory Appl. Categ. 10, 20 (2005)

9. Lurie, J.: $(\infty, 2)$-categories and the Goodwillie Calculus. 2009. arXiv: 0905.0462

10. Lurie, J.: Higher Topos Theory. Princeton University Press, Princeton (2009) 
11. Lurie, J.: Higher Algebra. http://people.math.harvard.edu/ lurie/papers/HA.pdf (2017) The author's webpage

12. Quillen, D.: Higher Algebraic K-Theory I Higher K-Theories Lecture Notes in Mathematics, vol. 341. Springer, Berlin (1973). https://doi.org/10.1007/BFb0067053

Publisher's Note Springer Nature remains neutral with regard to jurisdictional claims in published maps and institutional affiliations. 